\title{
iScout: An Intelligent Scout for Navigating Large Image Sets
}

\author{
Adam Weathermon ${ }^{a}$, Robert Rohling ${ }^{b}$ \\ ${ }^{a}$ Institute of Biomaterials and Biomedical Engineering, Univ. of Toronto, 4 Taddle Creek Road, \\ Rm. 407, Toronto, Ontario, M5S 3G9; \\ ${ }^{b}$ Dept. of Electrical and Computer Engineering, Univ. of British Columbia, 2356 Main Mall, \\ Vancouver, B.C., V6T 1 Z4
}

\begin{abstract}
A new intelligent software tool for PACS systems called 'iScout' has been developed that constructs and displays an overview of large series or studies before downloading the set of images to a PACS workstation. The overview consists of two orthogonal cross-section images that allow the user to select and download a subset of images, avoiding long delays that can occur while downloading hundreds or even thousands of images. The iScout also provides a navigational tool, allowing the user to click on anatomical regions and view the relevant slices, while displaying the anatomical location of the image currently being displayed by the PACS workstation software. The construction of an iScout can be done on either a workstation or a server with only minimal overhead that does not significantly affect the speed of loading. A working iScout tool has been integrated with multi-modality PACS workstation software (McKesson Medical Imaging Solutions), and it was found that the iScout can be generated on the workstation with a maximum added overhead of only 3.4 seconds while downloading a study containing 433 $512 \times 512 \mathrm{CT}$ images. The iScout is flexible and can generate scouts for virtually all types of CT and MR images, as well as 3D Ultrasound.
\end{abstract}

Keywords: PACS, workflow, scout, multiplanar reslice (MPR), iScout, priority downloading

\section{INTRODUCTION}

A challenging problem in PACS systems is the timely transmission and display of extremely large image series. This is due in part to the increasing capabilities of imaging modalities such as multi-slice spiral CT scanners. For example, a single scan from a modern CT scanner can generate over a thousand images with an uncompressed storage size of 524 Megabytes (assuming 1024 images with 512x512 pixels at 2 bytes/pixel and no overhead). This represents a significant amount of data, and transporting such an image series over a local area network can cause delays and unnecessary waiting time for radiologists. For example, the previously mentioned series would require over seven minutes to transport assuming full utilization of a $10 \mathrm{Mbps}$ network connection. Since full network bandwidth is unrealistic, actual delay times are longer for similar series, and will continue to increase as the scanning technology progresses.

Network delays in transporting large image series are significant because they result in poor workflow for radiologists. In other words, when the radiologist has selected a series to be viewed on a PACS review workstation, long delays in displaying the relevant series add up to a significant amount of time and these delays reduce the cost efficiency of the review process. Therefore, developing techniques that help speed up the downloading and navigating of large image sets improves workflow for radiologists, allowing them to view the greatest number of series in the least amount of time.

For example, it has also been shown that often a whole body scan can give all the required imaging information in the early stages of assessing trauma patients. Delays in downloading the images to the viewing workstation could therefore be significant in treatment of trauma patients. Another situation in which downloading time is an important issue is in the area of telemedicine, where a physician may access patient data over the Internet, with less bandwidth than local area networks.

An additional problem is that the size of some studies exceeds the physical memory of the workstation. Specifically, current 32-bit PC's can access 4Gbyte of memory of which only 2Gbyte can be reliably used to store the study. Therefore studies greater than 2 Gbyte cannot be loaded in their entirety and must be loaded one portion at 
a time. For these reasons, more intelligent strategies for image downloading and display are needed to allow timely and easy access to large data sets.

To address these issues, the Intelligent Scout Tool (iScout) has been developed to show an overview of a large series or study before downloading the entire set of images to a PACS review workstation. In this way, the user can select and download a subset of images in the series or study. This, in turn, avoids the long delays that occur when downloading hundreds or even thousands of images. An iScout image is composed of two multiplanar reslice (MPR) views of the study orthogonal to each other and the images.

A second motivation for the iScout tool is to provide a navigational tool to the user. Normally, images can be displayed in stack mode where a single image from the series is displayed and the user can navigate to different areas of the series by scrolling through the images (using the mouse wheel for example). The iScout tool displays an overview of the images (highlighting those that have been downloaded) with an indication of the currently displayed image on the stack. Instead of just scrolling to the desired region, the user can click on the desired anatomic region in the overview using the mouse, and the image that has been selected will be immediately displayed. Using the iScout tool in this way, the user can quickly navigate from one anatomical region to another by observing a global view of the set of images and their locations.

One additional advantage of using iScout occurs in situations where a traditional scout image may not spatially match a series of images because of patient motion. For example, in MRI a scout may be taken minutes after the initial scan. Any patient motion between these acquisitions means that a given physical location in the scout may not show the same anatomical feature as the same physical location in the series of images. This could have serious consequences in spine studies where mistakes over matching vertebrae can occur. Since the iScout is constructed from the series data directly, repositioning the patient for an additional scout image is not required, and there are no repositioning errors associated with the relative location of anatomical features in the scout and series images.

\section{METHODS}

\subsection{High Level Description}

The iScout is a new self-contained component that has been integrated with a multi-modality review workstation from McKesson Medical Imaging Solutions. iScout is written as an Active Template Library (ATL) COM object in Visual C++, and runs on a Windows platform. OpenGL is used for texture mapping and rending the iScout. The iScout is capable of displaying an overview of an image set and allows the user to select one or more images to download.

\subsection{Requirements for iScout}

The requirements for creating iScout images were as follows:

- The iScout must be created with a minimum of computational resources,

- iScouts must have low storage sizes relative to the storage size of a study,

- iScouts must conform to the DICOM standard,

- an iScout must depict an accurate overview of a given series,

- the iScout must contain patient orientation information

The requirements for viewing an iScout were as follows:

- iScouts must efficiently display all information in the iScout to the user,

- iScouts must occupy a small amount of screen real-estate,

- the user must be allowed to select quickly one or more images in the vicinity of desired part of the anatomy,

- iScouts must be shown according to a standard patient orientation. 
- iScouts can be created on server or on workstation. If the iScout is created on the workstation, the user can view the iScout while it is being built incrementally and still use the iScout for priority downloading.

\section{3. iScout Implementation}

The iScout Tool is composed of two components, the iScoutCreator and iScoutViewer. These components reside in a single COM object that can be run on any PACS server or workstation and can be controlled using a simple COM interface. The iScoutCreator is responsible for creating the MPR images and adding them to the study, while the iScoutViewer displays the iScout images in the standard viewing window. iScout images conform to the DICOM standard and are tagged as 'Secondary Capture' images.

\section{4. iScout Description}

An iScout is composed of an MPR image orthogonal to the scan images. Two MPR images are created by extracting the middle row and middle column of the images. The middle rows and columns of the images are then organized according to the image position and orientation information found in the DICOM header to create two orthogonal reslices of the study. Any gaps between images in the series are filled by interpolation so that all portions of the iScout are shown in the correct position, orientation and scale. By using the middle row and column of pixel data, it is easy to create the iScout images with minimal computational cost. These two orthogonal reslices of the study represent a visual summary of the entire series that can be used for navigating to different locations in the study. The iScout images are DICOM compatible for transmission, storage, and printing. Additional features supported in iScoutViewer are described below.

\section{Window/Level Control}

iScoutViewer supports window/level control by using the right mouse button, similar to the standard interface of the review workstation. The iScout also supports receiving window/level information from the PACS software to synchronize the window/level with other images on the screen. For example, if the user adjusts window/level in another window, the iScout will be displayed with these settings. The window/level capabilities are shown in the sequence of iScout images in Figure 1.

\section{Context Information}

As shown in Figures 1, 2, and 3, a rectangle wireframe 'surrounding' the iScout image indicates the orientation and location of the image currently being displayed in the PACS display window. When a user clicks on a region of the iScout, the image selected in the iScout is brought to the top of the stack by the workstation software. In this way, the iScout conveys context information about which anatomical region is being viewed in the series, and the radiologist can navigate to different regions of the series easily by clicking in iScoutViewer.

\section{Downloading Status Information}

The chits on the right hand side of the iScout show the images that have been downloaded to the PACS server while the study is being downloaded. For example, Figure 3 shows an iScout being created on the workstation while the study is being downloaded (note that this operation would already be complete if the iScout MPR images are created on the server). Notice that the images already downloaded are processed and their middle rows and columns are added to the iScout image. Gaps or black lines indicate images that are not yet loaded. The color of the chits indicates the number of images downloaded in that section of the series. The number beside the first chit indicates that the chit corresponds to a group (or chunk) of images, instead of one chit per image.

\section{Priority Downloading}

A radiologist can select a subset of images from a study for priority downloading by clicking on a region of the scout image in iScoutViewer while the study is being downloaded. Images that are selected for priority 


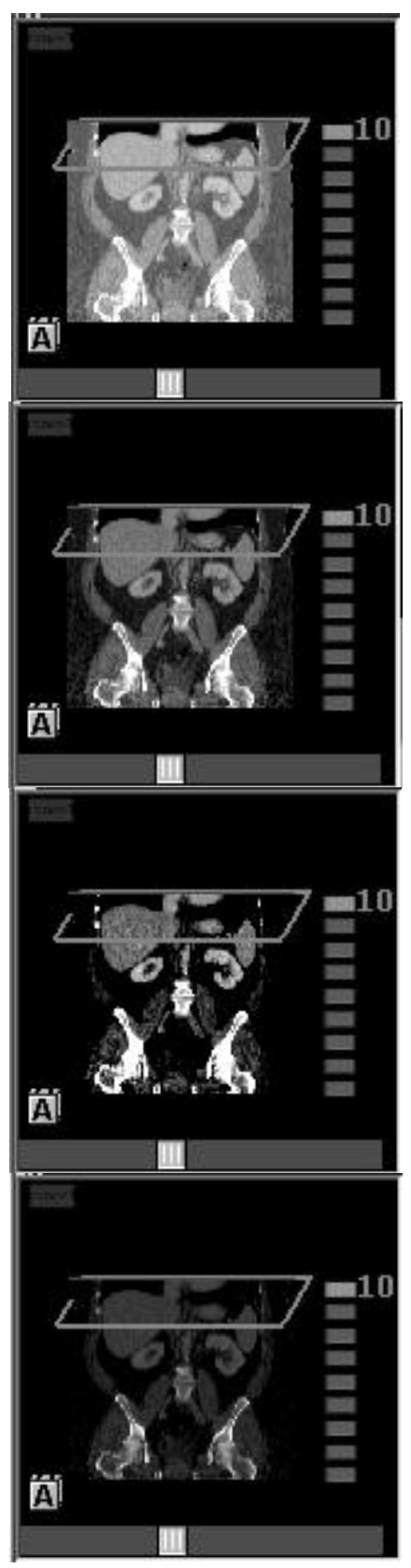

Figure 1

downloading are downloaded immediately after the request has been made - immediately after the current image being downloaded is finished. When an image is selected for priority downloading, iScoutViewer also determines the ten nearest images to the selected image and requests those images after the first image is downloaded. Therefore, the region of interest selected by the radiologist will be downloaded first for viewing. 


\section{Rotation Slider}

The square slider at the bottom of the iScoutViewer window allows the user to rotate the view of the iScout and select the other orthogonal MPR image to be displayed.
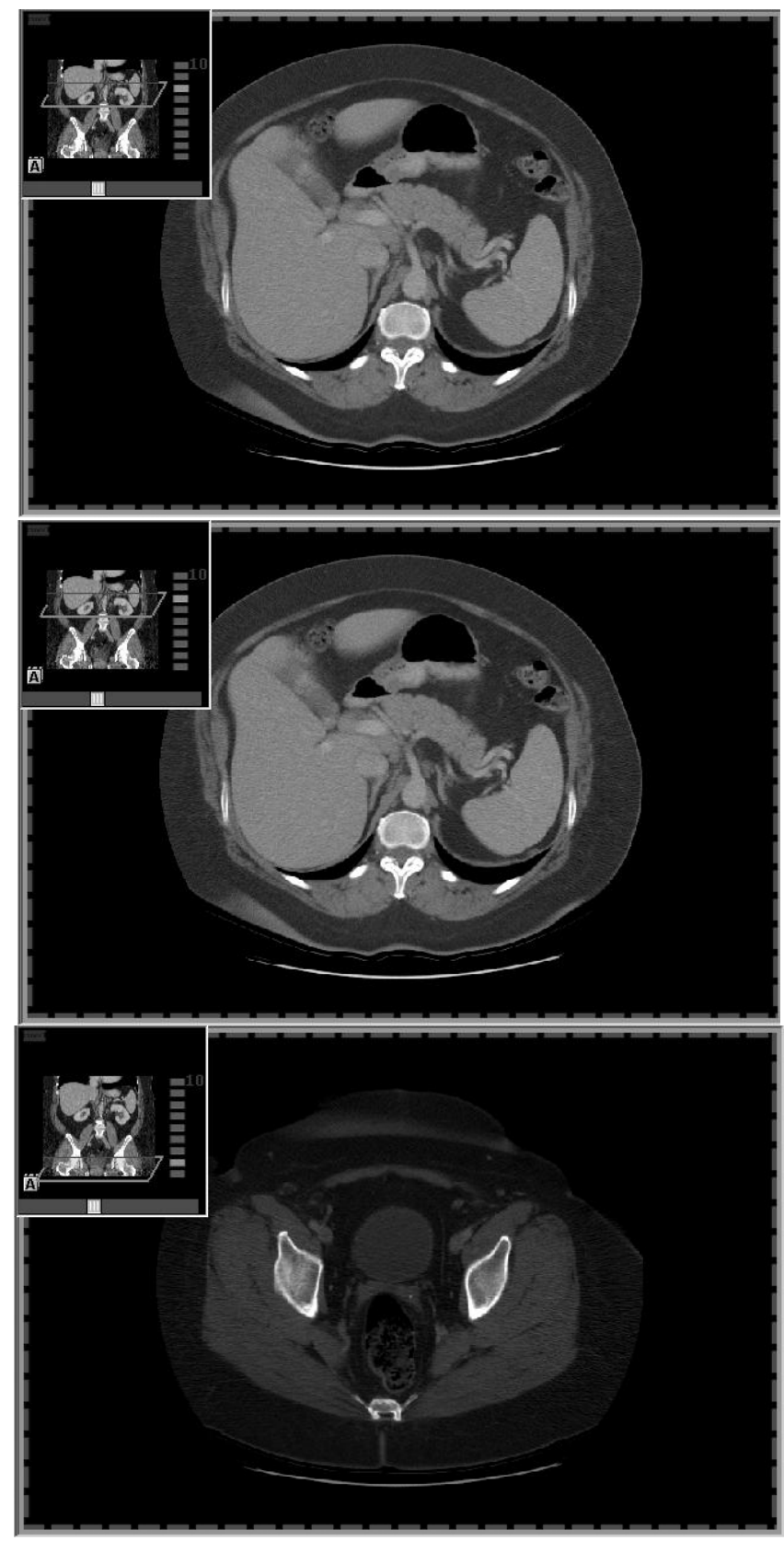

Figure 2 
The patient orientation cube in the lower left-hand corner shows the patient orientation of the iScout view, and rotates in real time when the slider is moved to show the other orthogonal image.

\subsection{How an iScout is Created}

The following event sequences describe how an iScout is generated and used by a radiologist when viewing a study.

\section{Event Sequence : Generation and Usage of an iScout}

- A study is sent from the imaging modality to the PACS server. iScoutCreator (running in the iScout Tool COM component on the server) creates an iScout image for the study containing both image and patient orientation information, and adds the iScout image to the study stored on the server.

- A radiologist sitting at a PACS review workstation selects the study. The workstation begins downloading the study from the server over the network.

- The iScout is the first image sent by the server and received by the PACS review workstation. The iScout is displayed immediately by iScoutViewer while the rest of the study is received one image at a time. The radiologist can use the iScout to select images for priority downloading by clicking on an anatomical region of the scout in iScoutViewer. The selected image and images nearby the selected image in the study are downloaded next. In other words, the iScout determines the ordering of the images to be downloaded by assigning images near the anatomical region of interest a high priority, and images far from the region of interest a low priority.

- As the images are downloaded and displayed, the user can also navigate to different anatomical locations in the study using the iScout by clicking on the region of interest. The iScout is displayed in a picture-inpicture style (shown in Figures 2 and 3), and the image being displayed on the review workstation is also indicated on the iScout as the wireframe rectangle.

- Once the entire series is downloaded, the user can continue to use the iScout to navigate the image series or close the iScout view if it is not needed further.

A variation on this protocol replaces iScout generation on the server with iScout generation on the workstation itself. In this scenario, the main difference is that the first time a study is downloaded, the iScout is not available and is instead created "on the fly" as the study is being downloaded. After the downloading is complete, the finished iScout is added to the PACS server, and further downloads of the series occur as described in the event sequence above. After the initial implementation of the iScout Tool, it was found that the iScout can be generated with minimal overhead, so future implementations of iScout Tool will perform the iScout creation on the server. The conclusions of the experiments are found to be the same for both protocols.

\section{RESULTS}

A working iScout has been created and integrated with a multi-modality PACS review workstation. Screen shots are shown in Figures 1,2, and 3. Figure 2 shows the radiologists view of an image in a study with the iScout shown in the upper left corner. Note that the iScout displays a reconstructed overview image of the series, and shows the location of the image currently being viewed, and has an orientation box in the lower left hand corner. Figure 1 shows a larger view of only the iScout window.

The iScout interface features two orthogonal MPR views of the image series (the sagittal plane being shown in the screenshots), adjustable window/level capability, patient orientation display, the ability to select images for viewing 


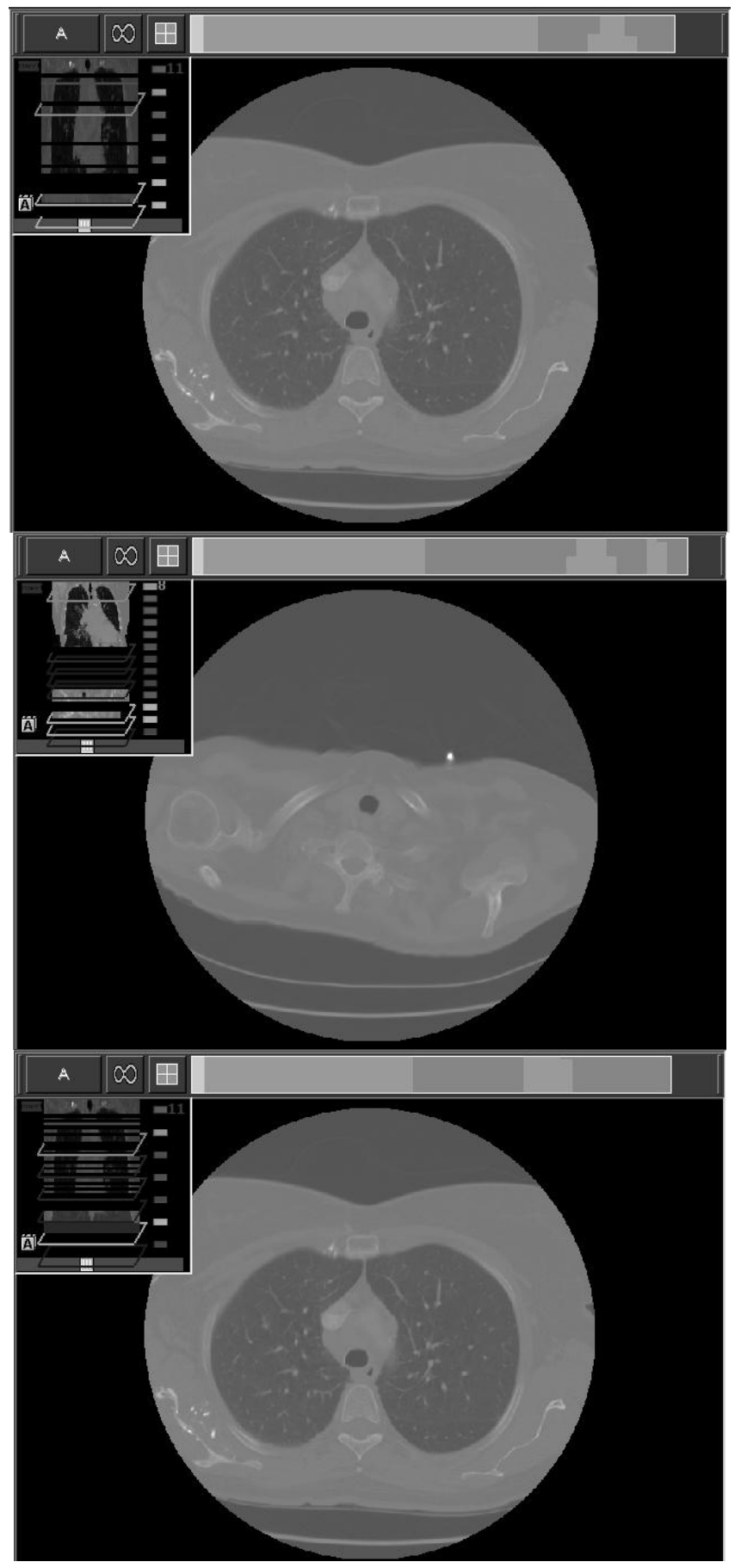

Figure 3

and downloading, and 3D rotation of the iScout. The user also has the capability of selecting groups of images from a particular spatial location, as well as selecting groups of images for priority downloading.

To measure the time needed to download an image of interest with and without iScout, five CT studies of different size were downloaded from a PACS server to a PACS review workstation over a local area network. Artificial network delays were added to simulate network transmission with increased network traffic since the testing was 
done on an in-house local area network with no network traffic. While varying the network delay, the average time (averaged over 30 trials) to load a random image from the five studies was recorded. This was assumed to be the middle image (for example, in the study containing 378 images this would correspond to the $189^{\text {th }}$ image). As well, the average time needed to select and receive an image from the same studies using the iScout tool was observed. When using iScout, the loading was measured assuming the radiologist would spend 15 seconds viewing the iScout and selecting the image. The results of these tests are shown in Figures 4 and 5. From the intersection of the curves shown in a figure, it is possible to estimate the size of study that would benefit from the priority downloading feature of iScout with a given network bandwidth.

The most dramatic results are for the large study containing 1028 images. For example, using an artificial network
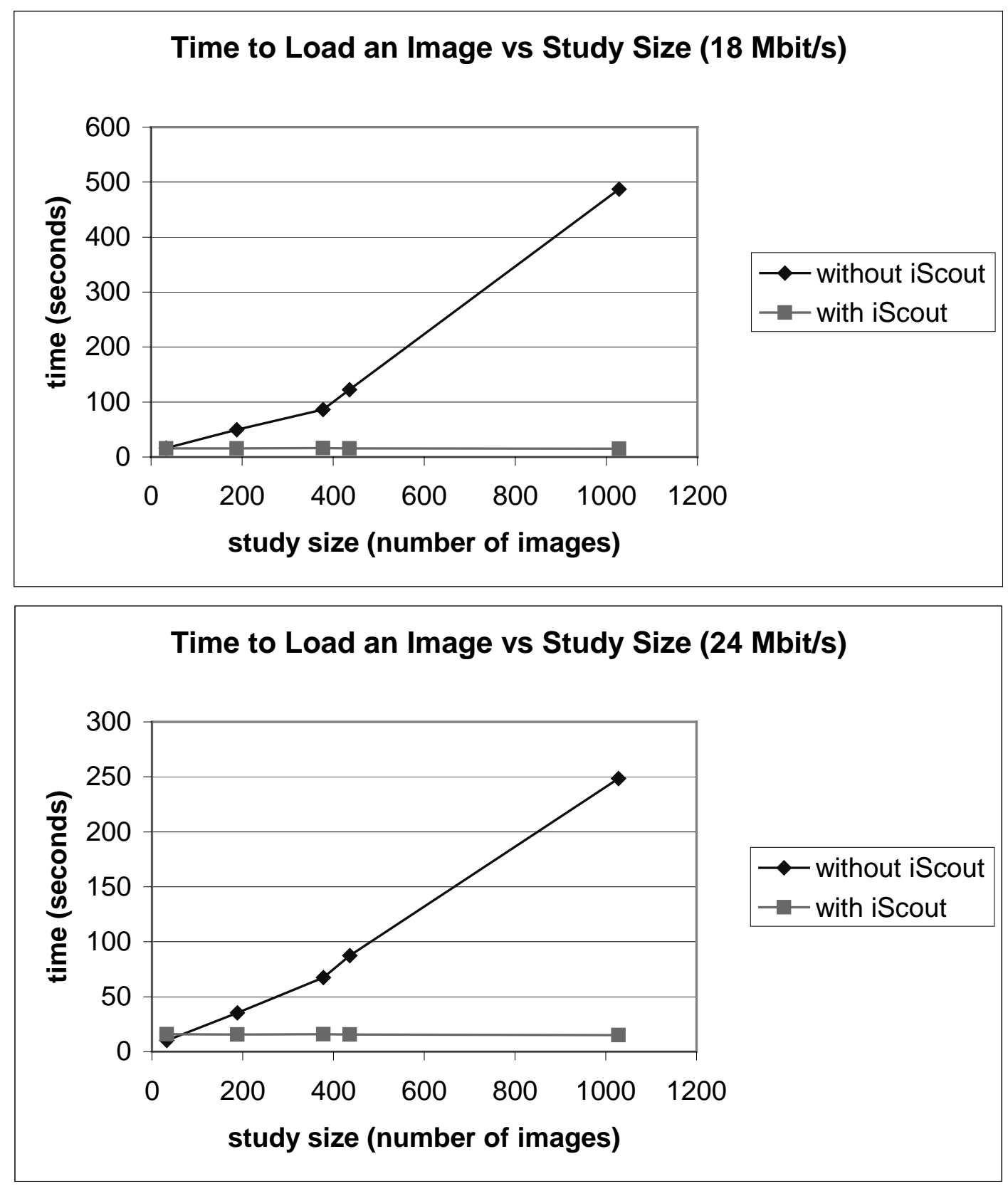

Figure 4 

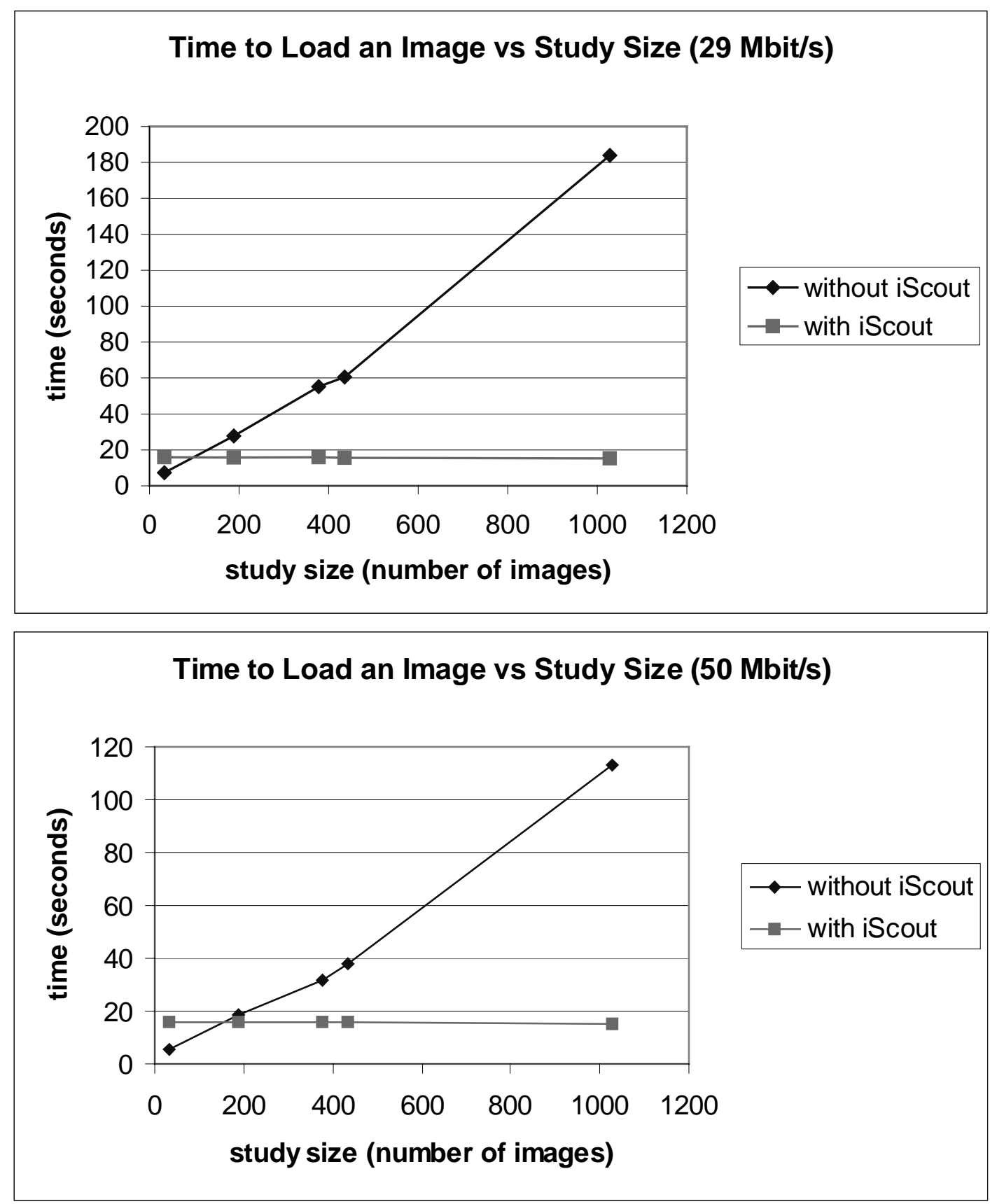

Figure 5

delay of $250 \mathrm{~ms}$, the corresponding network bandwidth is calculated to be approximately 24 Megabits/s based on the time to load a study and the size of the study. At this network speed, the mean loading time for a random image without iScout is 248.5 seconds, and using iScout the mean loading time is 16.1 seconds (assuming 15 seconds before the image is selected for priority downloading). In this simulation the image of interest is loaded 3.87 minutes faster with iScout. Of course, if the radiologist took more or less time to select images for priority downloading, this would affect the vertical position of the line in Figures 4 and 5. 
In simulations using the study containing 378 images, the maximum time savings using iScout was 1.4 minutes, which shows that iScout is useful for moderate sized datasets under the network conditions tested.

Another concern in developing the iScout was the computational burden of creating an iScout. To measure the processing overhead, the average (averaged over 50 trials) total time to load the test studies was measured and compared with the average total time to load the same studies while creating an iScout on the workstation "on the fly" as the data is being received. The data from these tests is shown in Figure 6. It was found that the average measured time overhead of using iScout ranged from 5.0 to 20.6 percent. For the largest study (1028 images), the average overhead was $7.9 \%$ or just over seven seconds. Therefore, the process of creating an iScout can safely be performed on the server without significant added processing.

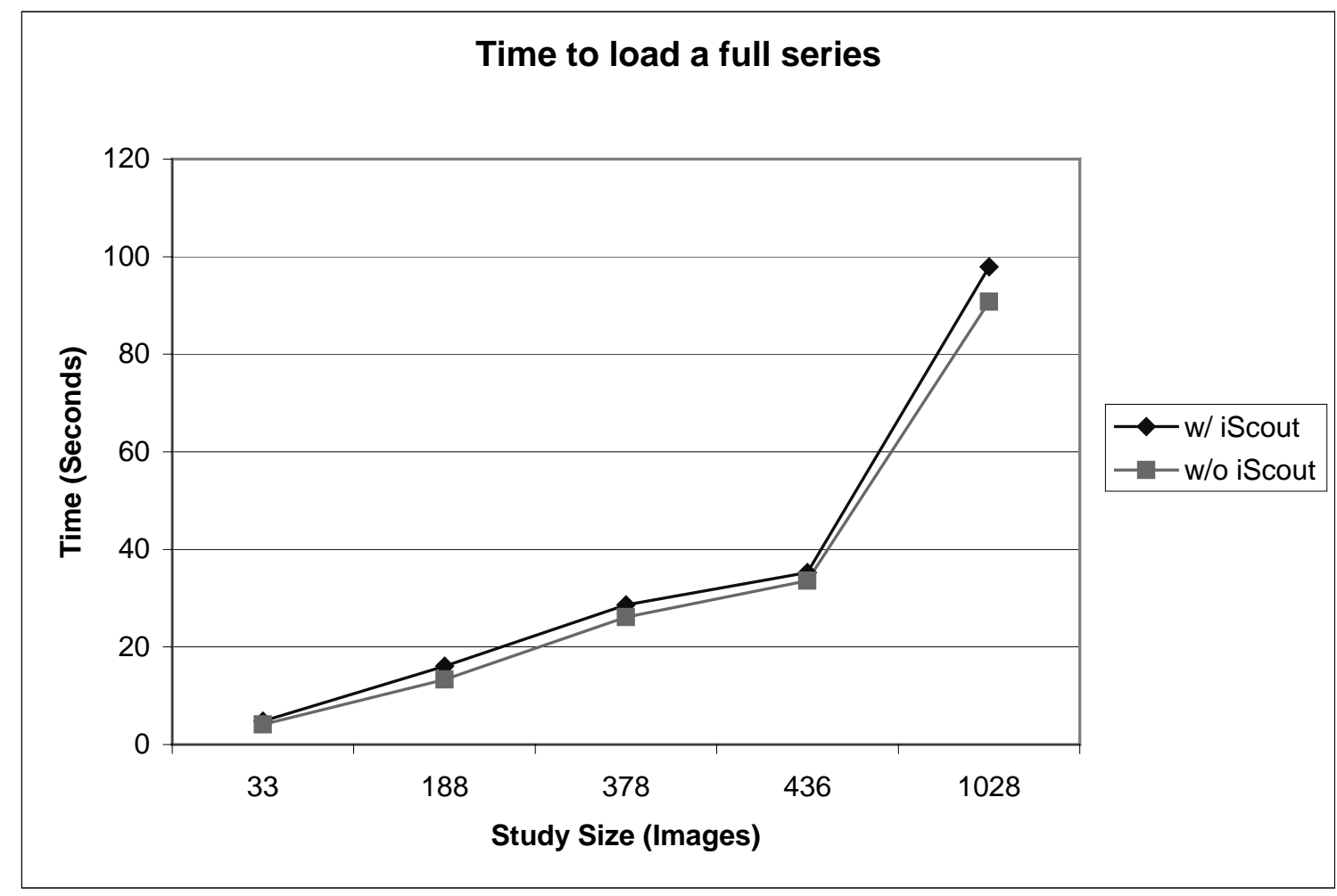

Figure 6

\section{DISCUSSION}

Although it was expected that the iScout would be primarily used for very large datasets on average to slow networks, our simulations showed that iScout is useful for even moderate-sized datasets on networks with moderate bandwidth. Tests also show the feasibility of using the iScout as a general-purpose tool for navigating the dataset once it has been loaded. The advantage of using iScout to navigate a large image series is that images can be selected for viewing by pointing to the desired anatomy on the overview images.

An iScout composed of two orthogonal cross-sections of a study can be generated quickly and depicts anatomy well. The construction of an iScout can be done on either a workstation or a server with only minimal overhead that would not affect the speed of loading. Using iScout to select images for priority downloading allows the user to select the correct image easily. The iScout is flexible and can generate scouts for virtually all types of CT and MR images, as well as 3D Ultrasound. The iScout has an intuitive interface and requires little training to operate. 
Overall the iScout addresses the need for new tools to handle the growing frequency of very large image sets that can be difficult to navigate efficiently and cause long delays during loading.

\section{ACKNOWLEDGEMENTS}

The authors would like to thank the McKesson staff - such as Len Grenier, Warren Edwards, Allan Noordvyk, David Branson, Mahmoud Ramze-Rezaee and the Emerald team - for valuable advice on this research. We also thank Jonathan Tsui for his contributions to the coding, debugging, and testing of the iScout software. 